\section{Carol-Nachman prize for rheumatology}

Professors J Sieper and J Braun from Berlin have been honoured with the Carol-Nachman prize for rheumatology of the city of Wiesbaden. The Carol-Nachman prize is the most prestigious international award in rheumatology. They received the prize for their work on the role of $\mathrm{T}$ cell cytokines in the pathogenesis of arthritides and as a possible target of immunomodulating treatments. Sieper and Braun studied the different reactivity of T helper 1 (Th1) and Th2 lymphocytes in various forms of arthritides, including reactive arthritis, Lyme arthritis, rheumatoid arthritis, ankylosing spondylitis, and osteoarthritis.

They found that the low Th1 response of the synovial membrane in patients with reactive arthritis and the high Th2 response in patients with Lyme arthritis is inadequate for an effective elimination of bacteria and a possible cause of bacterial persistence in these disorders. In addition, they described by flow cytometric analysis of synovial lymphocytes a high frequency of $\mathrm{T}$ cells specific for different bacterial antigens in the joint. In an open therapeutic study with monoclonal tumour necrosis factor blockers they showed that patients with active ankylosing spondylitis had a good anti-inflammatory response.

Professor Claude Bennett from Birmingham (USA) has been awarded the Carol-Nachman medal for his life's work and for his continuous support of young investigators in rheumatology.

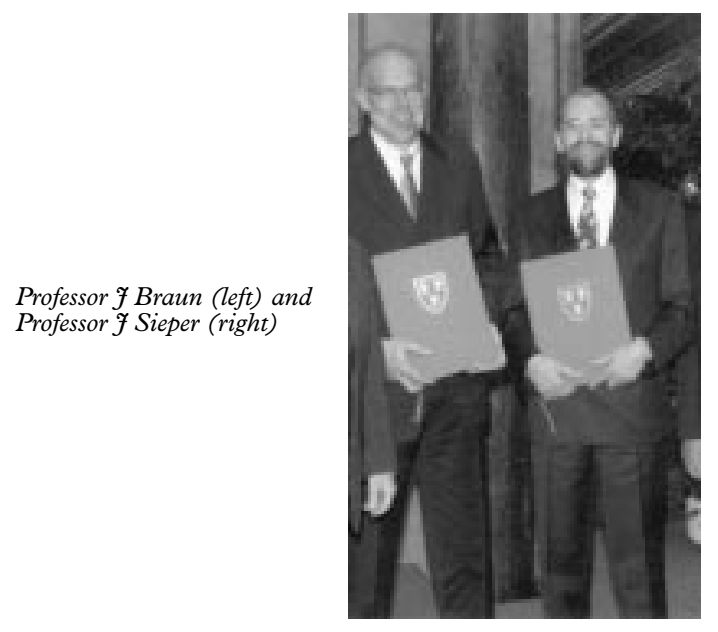

\section{Forthcoming events}

\section{In 2000}

1-4 Sep EULAR course: The Clinical Assessment of Joints and Spine, Frederiksberg, Denmark Registration before 15 August 2000

Further details given in Ann Rheum Dis 2000;59:512

Contact: Henning Bliddal, email: henning.bliddal@fh.hosp.dk

7-10 Sep 28th Scandinavian Congress of Rheumatology, Turku, Finland

Contact: Congress Office/Rheuma 2000, University of Turku, Lemminkäisenkatu 1418B, FIN-20520 Turku, Finland

Tel: +3582333 6342. Fax: +35823336410

Email: cescon@tkk.utu.fi
4-7 Oct 2nd International Congress on Spondyloarthropathies, Gent, Belgium

Contact: Medicongress, Waalpoel 28, B-9960

Assenede, Belgium

Tel: +32 934439 59. Fax: +329344 4010

Email: congresses@medicongress.com

29 Oct- 64th American College of Rheumatology

2 Nov AGM, Philadelphia, USA

Contact: Ronald F Olejko, Director of Conferences and Meetings, 60 Executive Park South, Suite 150, Atlanta, Georgia 30329, USA

Tel: +1 404633 3777. Fax: +1 4046331870

22-25 Nov Hellenic Congress on Rheumatology, Athens, Greece

Contact: Dr Grigoris Skarantavos, Hellenic Society for Rheumatology

Tel/fax: $00+301016545243$

Email: mail@ere.gr

7-9 Dec American Back Society Conference, Vancouver, Canada

Advances in spinal diagnosis and treatment for the 21 st century

Contact: Aubrey A Swartz, American Back Society, 2647 International Boulevard, Suite 401, Oakland, CA 94601, USA

Tel: +1 510536 9929. Fax: +15105361812

Email: info@americanbacksoc.org

Web site: www.americanbacksoc.org

In 2001

24-28 Mar 6th International Conference on Systemic Lupus Erythematosus, Barcelona, Spain

Contact: Secretariat: Viajes Iberia Congresos, Diagonal, $5234^{\circ} 2^{\mathrm{a}}$, 08029 Barcelona, Spain Tel: +34 93495 5306. Fax: +34 934051390 Email: congresos@v-iberia.com

4-5 May International Meeting on Gene/Cell Therapy in Osteoarticular Diseases, Montpellier, France

Contact: Dr Christian Jorgensen, Service d'Immuno-Rhumatologie, CHU Lapeyronie, 34295 Montpellier cedex 5, France

Fax: (33) 0467337798

Email: jorgens@montp.inserm.fr

13-16 Jun Annual European Congress of Rheumatology, Prague, Czech Republic

Contact: Fred Wyss, Executive Secretary, EULAR, Witikonerstrasse 15, CH-8032, Zurich, Switzerland

Tel: +411383 9690. Fax: +4113839810

Email: eular@bluewin.ch

Web site: www.eular.org

26-31 Aug XXth International League of Associations for Rheumatology, Edmonton, Canada

Contact: Oksana Kornilova, ILAR 2001 Marketing Coordinator

Tel: +1 8005353330 (Canada), +1 800642 2515 (US), + 12019475545 (other). Fax: + 12019478406 